\section{(1) \\ CrossMark}

\title{
New organisation for follow-up and assessment of treatment efficacy in sleep apnoea
}

\author{
Monique Suarez-Giron ${ }^{1}$, Maria R. Bonsignore $\mathbb{C}^{2,3}$ and Josep M. Montserrat ${ }^{1,4}$ \\ Affiliations: 'Unitat del Son, Servei de Pneumologia, Hospital Clinic, Barcelona, Spain. ${ }^{2}$ PROMISE Dept, \\ University of Palermo, Palermo, Italy. ${ }^{3}$ Institute of Biomedicine and Molecular Immunology, National Research \\ Council (CNR), Palermo, Italy. ${ }^{4}$ IDIBAPS, Facultat de Medicina, Universitat de Barcelona, CIBER de \\ Enfermedades Respiratorias, Madrid, Spain.
}

Correspondence: Maria R. Bonsignore, PROMISE Dept, University of Palermo, Piazza delle Cliniche 2, 90100 Palermo, Italy. E-mail: mariarosaria.bonsignoredunipa.it

@ERSpublications

Telemedicine could improve the management of sleep apnoea and other breathing disorders during sleep. http://bit.ly/2XPoPW1

Cite this article as: Suarez-Giron M, Bonsignore MR, Montserrat JM. New organisation for follow-up and assessment of treatment efficacy in sleep apnoea. Eur Respir Rev 2019; 28: 190059 [https://doi.org/10.1183/ 16000617.0059-2019].

ABSTRACT Obstructive sleep apnoea (OSA) is a highly prevalent disease, and there is an increased demand for OSA diagnosis and treatment. However, resources are limited compared with the growing needs for OSA diagnosis and management, and alternative strategies need to be developed to optimise the OSA clinical pathway. In this review, we propose a management strategy for OSA, and in general for sleep-disordered breathing, to be implemented from diagnosis to follow-up. For this purpose, the best current options seem to be: 1) networking at different levels of care, from primary physicians to specialised sleep laboratories; and 2) use of telemedicine. Telemedicine can contribute to the improved cost-effectiveness of OSA management during both the diagnostic and therapeutic phases. However, although the technology is already in place and different commercial platforms are in use, it is still unclear how to use telemedicine effectively in the sleep field. Application of telemedicine for titration of positive airway pressure treatment, follow-up to improve compliance to treatment through early identification and solution of problems, and teleconsultation all appear to be promising areas for improved OSA management.

\section{Introduction}

Obstructive sleep apnoea (OSA) together with insomnia are the most common sleep disorders [1, 2]. OSA is secondary to complete or partial airway obstruction caused by recurrent pharyngeal collapse during sleep, producing loud snoring or choking and frequent awakenings [3-5]. OSA may markedly affect quality of life and can be a risk factor for cardiovascular, metabolic and neurologic diseases, among others $[6,7]$. General measures, such as the treatment of obesity, exercise, avoidance of alcohol and sedatives, and sufficient amount of habitual sleep are important in OSA treatment but are often insufficient, and continuous positive airway pressure (CPAP) remains the optimal treatment option [8]. Increased knowledge of OSA by general practitioners and the general population has heightened the demand for consultations with a specialist. Over the past two decades, with the increasing prevalence of obesity, the number of patients diagnosed as suffering from OSA has increased drastically and will probably further increase over the coming years [2]. However, this increase in demand has not been accompanied by

Received: 25 May 2019 | Accepted after revision: 16 July 2019

Provenance: Commissioned article, peer reviewed

Copyright CERS 2019. This article is open access and distributed under the terms of the Creative Commons Attribution Non-Commercial Licence 4.0. 
strategic changes to make cost-efficient diagnosis, treatment or follow-up of patients with OSA. Therefore, there is a pressing need to improve OSA management.

All the problems listed above highlight the need for a change. To deal with these difficulties, and in view of the speed of current global change, continuous linear improvement needs to be developed in a short time [9]. Probably, the most important factors to be considered are the different medical levels involved in the clinical management of OSA, and the increasing availability of information and communications technologies.

In this review, we propose tentative management strategies for sleep-disordered breathing to be implemented from diagnosis to follow-up. As mentioned previously, a network of functioning and telemedicine techniques seem to be the best options, possibly generating a cost-effective personalised form of care provision [10-16]. There are a few points to be considered. 1) When a disease is common, all medical levels must be involved, from primary care, physicians and nurses, to reference (fully equipped, multidisciplinary teams or sleep specialists) and nonreference hospitals (able to perform simplified studies). 2) Sleep breathing disorders are not restricted to OSA. While uncomplicated OSA can be controlled by primary care medicine, other breathing disorders such as hypoventilation syndromes requiring noninvasive mechanical ventilation will likely become highly relevant in respiratory medicine in the near future. 3) The concept of patient-centred medicine has to be considered in all the different steps or procedures regarding sleep-disordered breathing. However, other sleep disorders, such as insomnia or restless leg syndrome, or nonsleep-related diseases such as obesity or depression are often associated with sleep-disordered breathing and require comprehensive patient management [17, 18]. 4) At present, technology has and will have even more importance in the management of sleep-disordered breathing in both diagnosis and follow-up. However, the technology should be simple, easy to use, reliable and, above all, transparent; it should not be a "black box". The better a technology is understood, the better it will be used $[19,20] .5)$ Other important aspects are the relationship with the administration, industry and other professionals. There is also a changing perspective regarding sleep, as it is currently considered at least as important as exercise or diet in general health. Therefore, healthy sleep is an important aspect to consider in the management of patients with OSA [21].

The network system, as its name implies, requires a close interrelationship between its medical components at each of the different clinical levels, with each piece knowing its hierarchy, influence area, communication channels, and common action guidelines and channelling systems. The keys to the successful operation of the network are autonomy, information, training and communication. This model allows optimisation of existing resources, making them available to the entire network and optimises priorities based on preference and complexity. Different groups have considered a greater role for primary care physicians, nurses and other healthcare professionals in the management of OSA. Ideally, this uses a hub-and-spoke model, in which OSA is predominantly managed in the community, with support for patients with more complicated or treatment-resistant symptoms provided in a sleep medicine centre [22-27].

Telemedicine offers the tools necessary to reach patients located in rural areas with health professional shortages. The use of telemedicine technologies that combine direct point-to-point sleep tests, data transmission, automatic CPAP systems and videoconference can help to establish diagnostic and therapeutic strategies for OSA from remote locations. These can be managed by a central sleep unit, maintaining an appropriate cost-effectiveness ratio, providing the possibility of avoiding travelling by the patients [28] and a reduction in lost productivity from work absenteeism [29]. Nonetheless, healthcare professionals providing telemedicine services should have the necessary education, training and orientation to ensure they possess the necessary competencies for the safe provision of quality health services, as well as a systematic quality improvement and performance management process that complies with organisational, regulatory or accrediting requirements for outcome management [30].

In addition, simplified devices are very useful in a large number of patients [31-37]; however, not all procedures can be applied universally because of problems in some healthcare environments or for other reasons. Other procedures currently applied are: 1) telemedicine management of sleep; and 2) studies performed over several days $[37,38]$. Until procedures are fully standardised in clinical care pathways and shown to be the most appropriate, it seems reasonable to follow different rules based on healthcare levels and severity of disease. The following basic steps are proposed (figure 1). 1) Primary medicine: if after the differential diagnosis, OSA is suspected, basic general measures for treatment should be initiated. 2) If symptoms are severe and the patients do not respond to the basic measures recommended, or other sleep-related diseases are suspected, the patient should be referred for diagnosis and treatment initiation in the sleep centre, fully equipped and with a multidisciplinary team, or able to manage simple devices and noncomplicated cases. 3) After diagnosis in the sleep centre and a therapeutic recommendation made, patients require follow-up. If the patient care goes well (with or without CPAP), the patient should return 


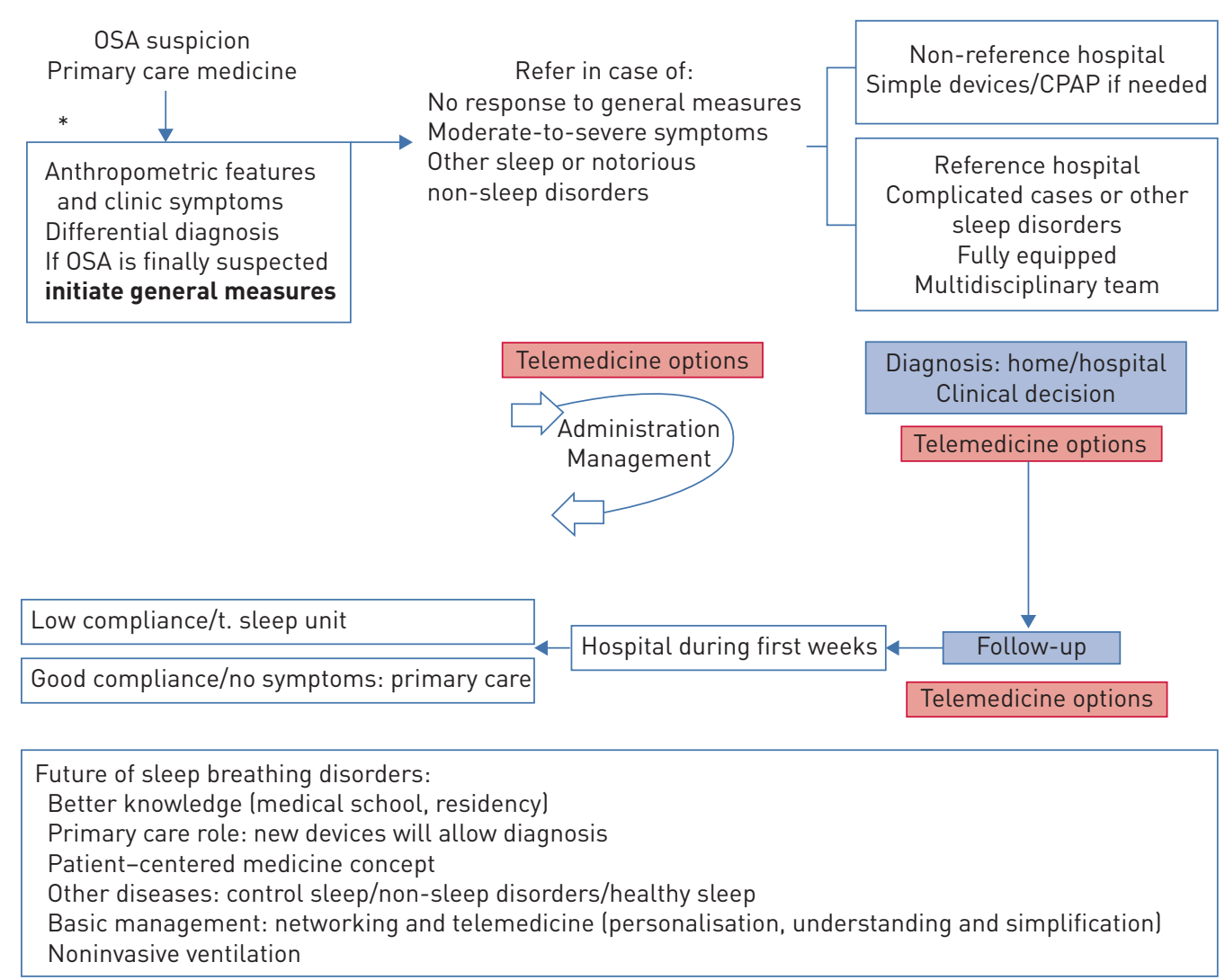

FIGURE 1 Overview of obstructive sleep apnoea (OSA) management. For OSA suspicion in primary care consider the following conditions: overweight, maxillo-mandibular abnormalities, heavy snoring and breathing pauses, nocturnal asphyxias and daytime somnolence. General measurements refer to: weight loss, exercise, sufficiently long sleep and avoidance of sedatives and alcohol. Differential diagnosis includes: inadequate sleep habits, depression with snoring, narcolepsy, hypoventilation syndromes, central sleep apnoea, restless leg syndrome, insomnia and substance abuse. CPAP: continuous positive airway pressure.

to the primary care physician with a clear indication of identifiable alert signs that may require referral back to the sleep centre. If CPAP is prescribed and compliance to treatment is good, lifestyle recommendations should be reinforced by the primary care physician. In case of poor compliance to treatment, patients should be reassessed and followed up by the sleep centre.

Telemedicine can have an important role in OSA management in the different steps as shown in figure 1, particularly during follow-up, as discussed later. Finally, two major points have to be considered and markedly improved: the role of primary care medicine and the implication of nursing staff, because in the near future with appropriate devices both of them can potentially contribute not only in the follow-up but also in the diagnosis with the implementation of simple devices. As already noted, adequate coordination between the different medical levels (networking) is imperative. To properly manage patients, personalised treatment, according to the entire spectrum of OSA phenotypes and associated comorbidities is a key point.

As far as sleep studies are concerned, there are two major types: full polysomnography (PSG; type 1 attended and type 2 non-attended) and home respiratory polygraphy (HRP; type 3 with at least four respiratory variables (flow, oximetry, band and position) and type 4 (just two respiratory variables) [39, 40]. PSG is considered to be the diagnostic gold standard; however, access to this procedure is limited because it requires special institutions with trained technicians and is relatively expensive overall. Organisations such as the American Academy of Sleep Medicine and the Australian Sleep Association recommend the management of OSA by HRP in patients with a high pre-istest OSA probability. A very short summary of the Australian Sleep Association recommendations follows: 1) A sleep study is only one component of the diagnosis. Clinical history and examination are as important and are complementary to the sleep study. The reference study is PSG at the sleep laboratory (type 1). 2) Type 2 studies have good diagnostic accuracy (to both "rule in" and "rule out" OSA) in selected patients and are an alternative to a type 1 study. 3) Home-based type 3 and 4 type sleep studies are useful to rule-in (but not rule out) OSA 
in patients with a high suspicion of OSA, or when combined with a validated sleep questionnaire. However, supervision by an accredited physician is recommended.

In general, a type 1 sleep study is recommended to diagnose sleep disorders, including sleep-disordered breathing; however, the diagnostic methodology will probably radically change in the near future due to the changes in the type of devices and information and communication technologies [41, 42] or development and use of new sensors [41]. New smartphones with sensors for ambient light, respiratory effort by built-in microphone, movement by built-in accelerometer and phone usage provide enough data to accurately estimate sleep and wake patterns. Moreover, the development of sleep medicine applications for sleep apnoea recognition with a continuous oximetry curve using an external sensor has been validated for screening children and adults providing an improved portable at-home screening tool, allowing multiple nights of monitoring. Furthermore, smart watches can carry sensors that pick up body signals directly without additional sensors being attached. However, evidence is scarce and although this could be considered easy, such new hardware and software systems require adequate validation [43]. Table 1 summarises the old and new diagnostic and titration procedures [41-43].

\section{CPAP follow-up}

Spanish guidelines for optimal CPAP follow-up recommend monitoring at 1-2 weeks, 1 month and 3 months after CPAP [44]. The next visits depend on the patient's characteristics, comorbidities, side-effects, residual symptoms, events detected and compliance. Since the early detection and treatment of side-effects is one of the most important points to control during the first weeks to ensure better compliance, the application of telemonitoring strategies seems a promising tool to achieve this goal [45]. The factors that predict successful long-term CPAP adherence identified in a recent trial in patients with moderate-to-severe OSA and cardiovascular disease (SAVE study) treated with CPAP were that the average hours of nightly CPAP use and side-effects at 1 month following the initiation of therapy [46] were consistent with results from previous studies in the OSA population [47, 48]. Other factors identifiable prior to commencing treatment such as initial problems (reported at auto-titration), recent life events and living alone have also been associated with lower CPAP use. Moreover, reporting problems after the first night of CPAP seems to be an important predictor of compliance [49].

\section{Telemonitoring CPAP and troubleshooting}

The telemonitoring of CPAP therapy has been used to improve compliance using different approaches of telemedicine but results have been inconsistent. Numerous providers have developed CPAP modems that enable the use of wired Ethernet cables, home Wi-Fi networks and Bluetooth connections to transfer data to a Cloud database. Technology continues to move forward by using cellular connection as the standard, with remote access to CPAP data by both providers and patients [50]. Access to this information likely benefits both parties. KUNA et al. [51] reported that when patients accessed their own data, CPAP adherence improved.

Moreover, randomised trials comparing telemonitoring follow-up versus usual care for CPAP therapy have shown a statistically significant improvement in CPAP use when CPAP data telemonitoring (compliance, leaks and apnoea-hypopnoea index) are used for clinician intervention management [52], associating with feedback via phone [53, 54]. HoEt et al. [55] showed that telemonitoring significantly reduced the delay to the first intervention for CPAP treatment (29 \pm 25 versus $47 \pm 30$ days), associated with the detection of problems by the telemonitoring system (39\% of patients). Additionally, compliance at 3 months was significantly better in the telemonitoring group (5.7 \pm 1.6 versus $\left.4.2 \pm 1.9 \mathrm{~h} \cdot \mathrm{night}^{-1}\right)$ [55]. Moreover, in a

\section{TABLE 1 Different diagnostic procedures}

\begin{tabular}{lrr} 
At present diagnosis & Near future & Diagnosis in the future \\
\hline $\begin{array}{l}\text { In-hospital or home PSG } \\
\text { HRP }\end{array}$ & $\begin{array}{r}\text { Home PSG/HRP remote transmission } \\
\text { Smartphone with different sensors }\end{array}$ & ALSHAER et al. [42] \\
& $\begin{array}{c}\text { Devices collecting data during several days } \\
\text { Contactless devices with data transmission }\end{array}$ & PENZEL SCHÖBEL et al. [43] \\
$\begin{array}{l}\text { Titration } \\
\text { PSG hospital } \\
\text { Home titration } \\
\text { Auto-CPAP treatment }\end{array}$ & Remote titration \\
\hline
\end{tabular}

PSG: polysomnography; HRP: home respiratory polygraph; CPAP: continuous positive airway pressure. 
recent trial conducted by HWANG et al. [56] that incorporated automated responses to the patient and education through a web platform (Tele-OSA), telemonitoring was useful in improving short-term compliance ( 3 months); however, at 1 year of follow-up it was observed that 3 months after the end of telemonitoring interventions compliance to CPAP was similar to that of patients who followed usual management, suggesting that the application of these strategies requires continuous application.

There are certain barriers when it comes to incorporating the use of CPAP telemonitoring into routine clinical practice, such as the use of different CPAP brands, the lack of standardisation of parameters used by different providers, preventing interoperability for healthcare professionals and the management of data within the existing electronic patient records. When using telecommunication systems, there is also the potential for network complications which should be adequately prevented and managed by a competent and fully available support service $[57,58]$.

\section{Teleconsultation}

Teleconsultation to support the clinical review of CPAP users is potentially useful and can be effective with regard to therapy compliance and user satisfaction, in addition to saving time taken from employment to attend specialistic visits at a sleep centre $[59,60]$. ISETTA and co-workers have performed two important studies $[45,59]$. In the first, 50 consecutive patients with OSA received a teleconsultation with a physician. Most patients were satisfied and $66 \%$ agreed that the teleconsultation could replace more than half of their CPAP follow-up visits [45]. In a second randomised study, 40 patients with OSA were divided into standard face-to-face training $(\mathrm{n}=20)$ or training via videoconference $(\mathrm{n}=20)$; subsequently they were blindly evaluated on what they had learned about OSA and mask placement [59]. Knowledge was comparable between groups, as was the performance of practical skills (mask and headgear placement, and leak avoidance) between groups [59].

Some concerns may be raised because of the limitation to perform a physical examination during teleconsultations. Despite that, patients with OSA could be adequately assessed without performing a physical examination that required the physician to be in the same room with the patient. Some elements of the examination, such as weight or blood pressure can be easily obtained by the nurse or primary care physicians [58].

Research is scarce regarding teleconsultation and telemonitoring; however, in a recent review by MURPHIE et al. [61], no safety concerns were raised. Two randomised trials showed improved CPAP compliance ( $n=19$ and $n=75)$; while two others $(n=114$ and $n=75)$ reported no differences between groups. Satisfaction was generally positively reported; one trial reported teleconsultation and telemonitoring as cost-effective. Therefore, well-designed and adequately powered trials are needed to establish whether this approach is a clinically effective and cost-effective option for CPAP users [61].

\section{Self-management and automated solutions}

With the continuous evolution of telemedicine applications, CPAP companies are also moving forward integrating features with expansion to follow-up with the aim of providing comprehensive management of

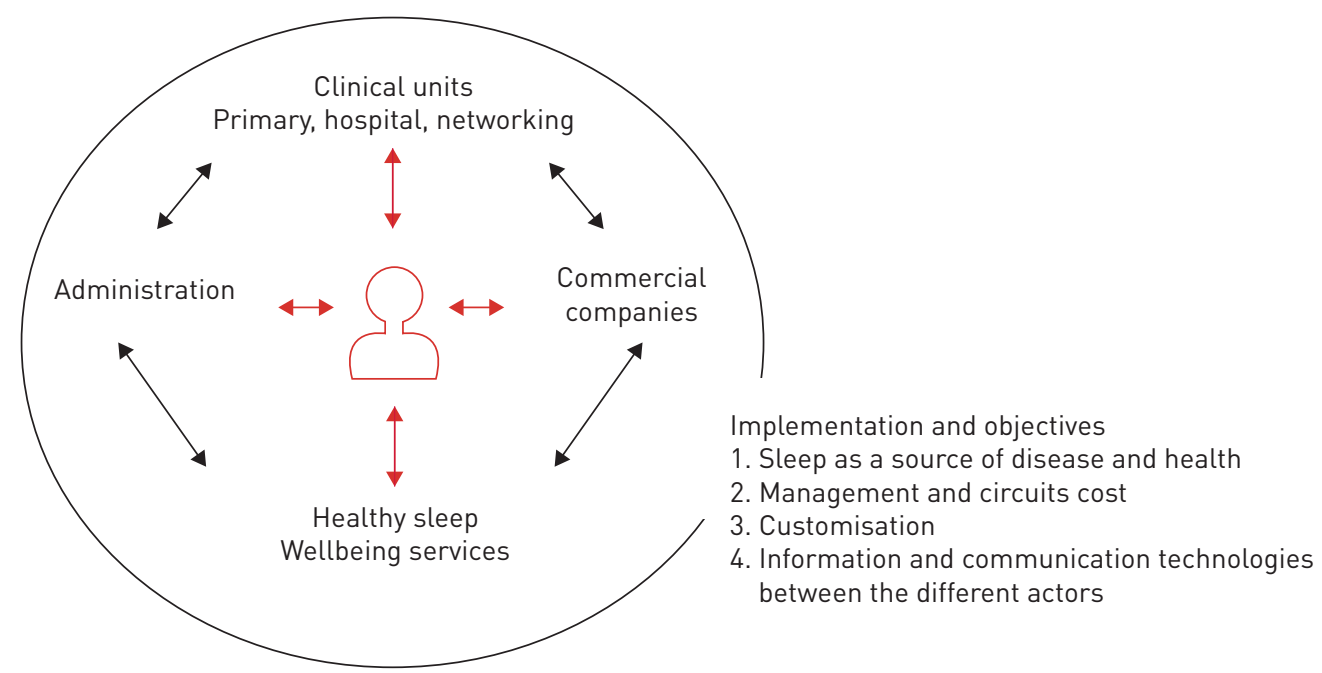

FIGURE 2 Strategies as a means of advancing patients health by improving patient care organisation, data management, diagnostic tools and follow-up support. 
OSA. Self-management platforms operate through mobile device applications (DreamMapper; Respironics, The Netherands, MyAir: ResMed, Australia), providing personal CPAP data in simple graphs to each patient, and also incorporating CPAP troubleshooting material. The data are transferred from the patients' CPAP to an online database, the same platform used by sleep professionals to access a comprehensive report of CPAP therapy data to monitor patients; and virtually all these patients have access to their CPAP data via an Internet browser (web portal) or a mobile device application. A retrospective review of 15000 patients in a database showed $78 \%$ of those who used SleepMapper were compliant at 90 days and used CPAP; however, it should be noted that a potential issue with this kind of retrospective uncontrolled data analysis is the possibility of confounding factors [50]. Moreover, for patients with comorbid insomnia, telemedicine also provides opportunities to exchange and automatically process sleep diaries and sleepwake data using smartphone applications, and to follow online programmes related to cognitive behaviour therapy [58, 62, 63]. Evidence also highlights the potential of mobile apps in improving symptom management and health outcomes through self-management interventions among those living with chronic diseases like OSA $[64,65]$. Mobile apps also represent an opportunity to reach patients routinely, and promote lifestyle modifications, such as nutrition and exercise [66], which can be of great use in OSA management.

\section{Summary}

The implementation of telemedicine in general practice is continuously growing, and both OSA and noninvasive mechanical ventilation belong to an area where much effort has been made to develop new strategies as a means of advancing patient health by improving patient care organisation, data management, diagnostic tools and follow-up support (figure 2). The American Academy of Sleep Medicine Task Force on sleep medicine supports telemedicine, while insisting on the importance of a clear understanding of its application and maintaining the clinical standards of care including all aspects of diagnosis and treatment [30]. Telemedicine applications can be useful to monitor and motivate patients with OSA treated with CPAP, by means of new mechanisms for supporting virtual visits and remote monitoring; however, further research into the impact, cost-effectiveness and outcomes is still needed for broad telemedicine enforcement. Basic concepts are simplification, understanding and personalisation of the processes. In addition, good coordination with companies, as well as appropriate training for both patients and professionals is imperative.

Conflict of interest: None declared.

Support statement: Funding was received from the Spanish Ministry of Economy and Competitiveness (PI14/00416 and PI17/01068). Funding information for this article has been deposited with the Crossref Funder Registry.

\section{References}

1 Bhaskar S, Hemavathy D, Prasad S. Prevalence of chronic insomnia in adult patients and its correlation with medical comorbidities. J Family Med Prim Care 2016; 5: 780-784.

2 Heinzer R, Vat S, Marques-Vidal P, et al. Prevalence of sleep-disordered breathing in the general population: the Hypno-Laus study. Lancet Respir Med 2015; 3: 310-318.

3 Dempsey JA, Veasey SC, Morgan BJ, et al. Pathophysiology of sleep apnea. Physiol Rev 2010; 90: 47-112.

4 Li Y, Orr J, Jen R, et al. Is there a threshold that triggers cortical arousals in obstructive sleep apnea. Sleep 2019; 42: zsz047.

5 Darquenne C, Elliott AR, Sibille B, et al. Upper airway dynamic imaging during tidal breathing in awake and asleep subjects with obstructive sleep apnea and healthy controls. Physiol Rep 2018; 6: e13711.

6 Lévy P, Kohler M, McNicholas WT, et al. Obstructive sleep apnoea syndrome. Nat Rev Dis Primers 2015; 25: 15015.

7 Lim DC, Pack AI. Obstructive sleep apnea: update and future. Annu Rev Med 2017; 68: 99-112.

8 Sullivan CE, Issa FG, Berthon-Jones M, et al. Reversal of obstructive sleep apnoea by continuous positive airway pressure applied through the nares. Lancet 1981; 1: 862-865.

9 Doarn CR, Merrell RC. Are you a disruptor and is anyone paying attention? Telemed J E Health 2015; 21: 319-320.

10 Aurora RN, Collop NA, Jacobowitz O, et al. Quality measures for the care of adult patients with obstructive sleep apnea. J Clin Sleep Med 2015; 11: 357-383.

11 Suárez M, Osorio J, Torres M, et al. Should the diagnosis and management of OSA move into general practice? Breathe 2016; 12: 243-247.

12 Chamorro N, Sellarés J, Millán G, et al. An integrated model involving sleep units and primary care for the diagnosis of sleep apnoea. Eur Respir J 2013; 42: 1151-1154.

13 Suarez-Giron MC, Isetta V, Masa JF, et al. Sleep breathing disorders: have we reached the tipping point? ERJ Open Res 2018; 4: 00172-2017.

14 Hernández L, Torrella M, Roger N, et al. Management of sleep apnea: concordance between non-reference and reference centers. Chest 2007; 132: 1853-1857.

15 Parra O, García-Esclasans N, Montserrat JM, et al. Should patients with sleep apnoea/hypopnea syndrome be diagnosed and managed on the basis of home sleep studies? Eur Respir J 1997; 10: 1720-1724. 
16 Montserrat JM, Sánchez-de-la-Torre M, Terán J, et al. Respiratory disorders during sleep: a new dimension for 2018. Arch Bronconeumol 2019; 55: 122-123.

17 Shelgikar AV, Durmer JS, Joynt KE, et al. Multidisciplinary sleep centers: strategies to improve care of sleep disorders patients. J Clin Sleep Med 2014; 10: 693-697.

18 Heatley EM, Harris M, Battersby M, et al. Obstructive sleep apnoea in adults: a common chronic condition in need of a comprehensive chronic condition management approach. Sleep Med Rev 2013; 7: 349-355.

19 Lugo V, Villanueva JA, Garmendia O, et al. The role of telemedicine in obstructive sleep apnea management. Expert Rev Respir Med 2017; 11: 699-709.

20 Farré R, Navajas D, Montserrat JM. Is telemedicine a key tool for improving continuous positive airway pressure adherence in patients with sleep apnea? Am J Respir Crit Care Med 2018; 197: 12-14.

21 Mukherjee S, Patel SR, Kales SN, et al. An official American Thoracic Society statement: the importance of healthy sleep. Recommendations and future priorities. Am J Respir Crit Care Med 2015; 191: 1450-1458.

22 Chai-Coetzer CL, Antic NA, Rowland LS, et al. A simplified model of screening questionnaire and home monitoring for obstructive sleep apnoea in primary care. Thorax 2011; 66: 213-219.

23 Chai-Coetzer CL, Antic NA, Rowland LS, et al. Primary care versus specialist sleep center management of obstructive sleep apnea and daytime sleepiness and quality of life. a randomized trial. JAMA 2013; 309: 997-1004.

24 Sánchez-Quiroga MÁ, Corral J, Gómez-de-Terreros FJ, et al. Primary care physicians can comprehensively manage sleep apnea patients: a non-inferiority randomized controlled trial. Am J Respir Crit Care Med 2018; 198: 648-656.

25 Tarraubella N, Sánchez-de-la-Torre M, Nadal N, et al. Management of obstructive sleep apnoea in a primary care vs sleep unit setting: a randomized controlled trial. Thorax 2018; 73: 1152-1160.

26 Kuna ST. Diagnosis and management of patients with obstructive sleep apnea in primary care. ready or not? Am J Respir Crit Care Med 2018; 198: 557-558.

27 Sánchez-de-la-Torre M, Nadal N, Cortijo A, et al. Role of primary care in the follow-up of patients with obstructive sleep apnoea undergoing CPAP treatment: a randomized controlled trial. Thorax 2015; 70: 346-352.

28 Coma-Del-Corral MJ, Alonso-Álvarez ML, Allende M, et al. Reliability of telemedicine in the diagnosis and treatment of sleep apnea syndrome. Telemed J E Health 2013; 19: 7-12.

29 Isetta V, Negrín MA, Monasterio C, et al. A Bayesian cost-effectiveness analysis of a telemedicine-based strategy for the management of sleep apnoea: a multicentre randomised controlled trial. Thorax 2015; 70: 1054-1061.

30 Singh J, Badr MS, Diebert W, et al. American Academy of Sleep Medicine (AASM) position paper for the use of telemedicine for the diagnosis and treatment of sleep disorders. J Clin Sleep Med 2015; 11: 1187-1198.

31 Mulgrew AT, Fox N, Ayas NT, et al. Diagnosis and initial management of obstructive sleep apnea without polysomnography: a randomized validation study. Ann Intern Med 2007; 146: 157-166.

32 Oliveira MG, Nery LE, Santos-Silva R, et al. Is portable monitoring accurate in the diagnosis of obstructive sleep apnea syndrome in chronic pulmonary obstructive disease? Sleep Med 2012; 13: 1033-1038.

33 Masa JF, Duran-Cantolla J, Capote F. Effectiveness of home single-channel nasal pressure for sleep apnea diagnosis. Sleep 2014; 37: 1953-1961.

34 Chai-Coetzer CL, McEvoy RD. The debate should now be over: simplified cardiorespiratory sleep tests are a reliable, cost-saving option for diagnosing obstructive sleep apnea. Am J Respir Crit Care Med 2017; 196 : 1096-1098.

35 Chai-Coetzer CL, McArdle N, McEvoy RD. Laboratory polysomnography or limited-channel sleep studies for obstructive sleep apnea. Ann Intern Med 2017; 167: 521-522.

36 Corral J, Sánchez-Quiroga MÁ, Carmona-Bernal C, et al. Conventional polysomnography is not necessary for the management of most patients with suspected obstructive sleep apnea. Noninferiority, randomized controlled trial. Am J Respir Crit Care Med 2017; 196: 1181-1190.

37 Guerrero A, Embid C, Isetta V, et al. Management of sleep apnea without high pretest probability or with comorbidities by three nights of portable sleep monitoring. Sleep 2014; 37: 1363-1373.

38 Fields BG, Behari PP, McCloskey S, et al. Remote ambulatory management of veterans with obstructive sleep apnea. Sleep 2016; 39: 501-509.

39 Rosen IM, Kirsch DB, Carden KA, et al. American Academy of Sleep Medicine Board of Directors. Clinical use of a home sleep apnea test: an updated American Academy of Sleep Medicine position statement. J Clin Sleep Med 2018; 14: 2075-2077.

40 Douglas JA, Chai-Coetzer CL, McEvoy D, et al. Guidelines for sleep studies in adults-a position statement of the Australasian Sleep Association. Sleep Med 2017; 36: Suppl. 1, S2-S22.

41 Han S, Kim J, Won SM, et al. Battery-free, wireless sensors for full-body pressure and temperature mapping. Sci Transl Med 2018; 10: eaan4950.

42 Alshaer H. New technologies for the diagnosis of sleep apnea. Curr Hypertens Rev 2016; 12: 48-56.

43 Penzel Schöbel C, Fietze I. New technology to assess sleep apnea: wireless, Smartphones and accessories. F1000Res 2018; 29: 413.

44 Lloberes P, Durán-Cantolla J, Martínez-García MÁ, et al. Diagnosis and treatment of sleep apnea-hypopnea syndrome. Spanish Society of Pulmonology and Thoracic Surgery. Arch Bronconeumol 2011; 47: 143-156.

45 Isetta V, Ruiz M, Farré R, et al. Supporting patients receiving CPAP treatment: the role of training and telemedicine. In: Barbé F, Pépin J-L, eds. Obstructive Sleep Apnoea. Sheffield, ERS Monograph 2015; 67: 280-292.

46 Chai-Coetzer CL, Luo YM, Antic NA, et al. Predictors of long-term adherence to continuous positive airway pressure therapy in patients with obstructive sleep apnea and cardiovascular disease in the SAVE study. Sleep 2013; 36: 1929-1937.

47 McArdle N, Devereux G, Heidarnejad H, et al. Long-term use of CPAP therapy for sleep apnea/hypopnea syndrome. J Am J Respir Crit Care Med 1999; 159: 1108-1114.

48 Popescu G, Latham M, Allgar V, et al. Continuous positive airway pressure for sleep apnoea/hypopnoea syndrome: usefulness of a 2-week trial to identify factors associated with long term use. Thorax 2001; 56: 727-733.

49 Lewis KE, Seale L, Bartle IE, et al. Early predictors of CPAP use for the treatment of obstructive sleep apnea. Sleep 2004; 27: 134-138.

50 Hwang D. Monitoring progress and adherence with positive airway pressure therapy for obstructive sleep apnea: the roles of telemedicine and mobile health applications. Sleep Med Clin 2016; 11: 161-171. 
51 Kuna ST, Shuttleworth D, Chi L, et al. Web-based access to positive airway pressure usage with or without an initial financial incentive improves treatment use in patients with obstructive sleep apnea. Sleep 2015; 38: 1229-1236.

52 Stepnowsky CJ, Palau JJ, Marler MR, et al. Pilot randomized trial of the effect of wireless telemonitoring on compliance and treatment efficacy in obstructive sleep apnea. J Med Internet Res 2007; 9: e14.

53 Fox N, Hirsch-Allen AJ, Goodfellow E, et al. The impact of a telemedicine monitoring system on positive airway pressure adherence in patients with obstructive sleep apnea: a randomized controlled trial. Sleep 2012; 35: 477-481.

54 Frasnelli M, Baty F, Niedermann J, et al. Effect of telemetric monitoring in the first 30 days of continuous positive airway pressure adaptation for obstructive sleep apnoea syndrome - a controlled pilot study. $J$ Telemed Telecare 2016; 22: 209-214.

55 Hoet F, Libert W, Sanida C, et al. Telemonitoring in continuous positive airway pressure-treated patients improves delay to first intervention and early compliance: a randomized trial. Sleep Med 2017; 39: 77-83.

56 Hwang D, Chang JW, Benjafield AV, et al. Effects of telemedicine education and telemonitoring on continuous positive airway pressure adherence. The Tele-OSA Randomized Trial. Am J Respir Crit Care Med 2018; 197: $117-126$.

57 World Health Organization. Telemedicine: opportunities and developments in member states: report on the second global survey on eHealth. Geneva, World Health Organization, 2010.

58 Verbraecken J. Telemedicine applications in sleep-disordered breathing: thinking out of the box. Sleep Med Clin 2016; 11: 445-459.

59 Isetta $\mathrm{V}$, León $\mathrm{C}$, Torres $\mathrm{M}$, et al. Telemedicine-based approach for obstructive sleep apnea management: building evidence. Interact J Med Res 2014; 3: e6.

60 Parikh R, Touvelle MN, Wang H, et al. Sleep telemedicine: patient satisfaction and treatment adherence. Telemed J E Health 2011; 17: 609-614.

61 Murphie P, Little S, McKinstry B, et al. Remote consulting with telemonitoring of continuous positive airway pressure usage data for the routine review of people with obstructive sleep apnoea hypopnoea syndrome: a systematic review. J Telemed Telecare 2019; 25: 17-25.

62 Ritterband LM, Thorndike FP, Gonder-Frederick LA, et al. Efficacy of an internet-based behavioural intervention for adults with insomnia. Arch Gen Psychiatry 2009; 66: 692-698.

63 Espie CA, Kyle SD, Williams C, et al. A randomized, placebo-controlled trial of online cognitive behavioral therapy for chronic insomnia disorder delivered via an automated media-rich web application. Sleep 2012; 35: 769-781.

64 Whitehead L, Seaton P. The effectiveness of self-management mobile phone and tablet apps in long-term condition management: a systematic review. I Med Internet Res 2016; 18: e97.

65 Tuckson RV, Edmunds M, Hodgkins ML. Telehealth. N Engl J Med 2017; 377: 1585-1592.

66 Elavsky S, Smahel D, Machackova H. Who are mobile app users from healthy lifestyle websites? Analysis of patterns of app use and user characteristics. Transl Behav Med 2017; 7: 891-901. 